\title{
LI. Bolometric investigations on the intensity of radiation by rarefied gases under the influence of electric discharge
}

\section{Knut Ångström}

To cite this article: Knut Ångström (1893) LI. Bolometric investigations on the intensity of radiation by rarefied gases under the influence of electric discharge , Philosophical Magazine Series 5, 35:217, 502-506, DOI: 10.1080/14786449308621422

To link to this article: http://dx.doi.org/10.1080/14786449308621422

曲 Published online: 08 May 2009.

Submit your article to this journal $[\pi$

Џll Article views: 3

Q View related articles $\llbracket$ 
fectly determinate except $\mathrm{T}_{1}$. If $\mathrm{CO}=a, \mathrm{CA}=b$, and $\mathrm{T}_{a}, \mathrm{~T}_{b}$ denote the tensions along $\mathrm{OB}, \mathrm{AD}$, we have

$$
\left.\begin{array}{l}
\mathrm{T}_{a}=\frac{\mathrm{A}}{a}-\frac{k \tan \alpha}{a^{2}} \\
\mathrm{~T}_{b}=\frac{\mathrm{A}}{b}-\frac{k \tan \alpha}{b^{2}}
\end{array}\right\}, \cdot . \cdot \cdot .
$$

from which it appears that either $\mathrm{T}_{a}$ or $\mathrm{T}_{b}$ may, if we please, be made zero, provided the other be properly determined.

The above results would also apply to a belt of a complete cone, bounded by two circular sections.

LI. Bolometric Investigations on the Intensity of Radiation by Rarefied Gases under the Influence of Electric Discharge. By KnUt Ångström*.

$\mathrm{NE}^{\mathrm{NE}}$ of the peculiar difficulties attending the quantitative determination of the amount of energy radiated by gases in vacuum-tubes is the extreme feebleness of its intensity. In his recent work in this field, executed at the Hochschule at Stockholm, Prof. Ångström attacked the problem by the bolometric method, which, although leaving something to be desired as regards sensitiveness, led to some important results. Another obstacle lay in the well-known difficulty of obtaining the gases in a state of such purity that the spectrum exhibited by the discharge through them in a vacuum-tube showed no foreign admixture, such as the carbon-bands seen whenever grease is used for joining surfaces, or where the flame touches in the process of soldering.

The discharge-tube used for the most careful experiments was thoroughly cleaned after soldering-in two electrometerterminals, and attaching two short lengths of tubing at right angles near each end. The latter were to receive the electrodes, whose construction required particular care. Into a short capillary tube a piece of platinum wire was introduced from one end and a piece of thoroughly cleaned aluminium wire from the other. - The tube was then heated so as to form an air-tight junction between the two wires, and was then fitted into a glass plate. After removing all grease the tube covering the aluminium wire was cut off', so that the latter acted as a perfectly clean electrode, and the glass plate holding it was fitted on to the short tube attached to the discharge-

* Abstract from Wiedemann's Annalen, No. 3,1893, by E. E. Fournier d'Albe, B.Sc., Royal College of Science. 
tube. The joints were made air-tight by means of sodium silicate, which proved to be a highly useful cement, and did not give rise to any impurities. Short lengths of tubing containing mercury were placed round the platinum wires to convey the current to the electrodes.

The behaviour of four gases only was investigated, viz. hydrogen, oxygen, nitrogen, and carbonic oxide. Hydrogen and oxygen were prepared by electrolysis of pure newlydistilled water acidulated with phosphoric acid. The nitrogen was obtained by passing pure air over heated copper-turnings reduced by hydrogen. Carbonic oxide was prepared by the reaction of sulphuric and oxalic acids, and purified by passing through caustic potash. In producing these gases all rubber tubes were dispensed with, and the different parts of the generating apparatus were soldered together.

The discharge-tube was connected through a Kundt glass spring and a set of cleaning-tubes to the tube used for introducing the gas, a mercury-valve, and the air-pump. The mercury-valve consisted of a U-tube communicating at the bottom with a long tube full of mercury. By varying the level of the mercury by means of a reservoir the valve could be opened and closed. The tube for introducing the gases corresponded in the main to Cornu's arrangement. A vertical glass tube is filled with mercury whose level can be varied by means of a reservoir connected through a flexible tube, as in the case of the mercury-valve. At a point some distance from the bottom is attached a capillary U-tube, the end of which, in the process of filling, is introduced into a small reservoir containing the gas. ILowering the mercury establishes a connexion with the discharge-tube through the drying-tubes, and on raising the level the gas is shut off from the atmosphere.

The current was furnished by a battery of 800 small Planté accumulators, regulated by means of a liquid resistance consisting of cadmium iodide dissolved in amyl alcohol, and measured by a dead-beat reflecting-galvanometer. The fall of potential in the discharge-tube was measured by a Mascart quadrant-electrometer.

'The bolometer used for the experiments consisted of two gratings cut out of tinfoil mounted in ebonite frames. These frames were placed one behind the other in a tube with double walls, the posterior one being protected from radiation by a small double screen. Four diaphragms were mounted in the tube in front of the gratings, to diminish air-currents. The grating absorbing the radiation occupied a circular space of 16 millim. diameter. It was blackened by precipitated 
platinum and smoke. The four branches of the Wheatstonebridge arrangement, of which the gratings formed two, had each a resistance of about $5 \mathrm{ohms}$. In order to be able to rapidly test the sensitiveness of the combination, a constant resistance was introduced as a secondary circuit into one of the branches. The opening or closing of this circuit usually made a difference of 75 scale-divisions. If not, the reading was reduced to that standard sensitiveness.

The bolometer was separated from the end of the dischargetube by a double screen with a perforation, inside which was suspended a small screen. This was quickly pulled up to expose the bolometer. The strength of current through the discharge-tube was measured by the galvanometer, the difference of potential within it by the electrometer, and the deflexion of the galvanometer in the bolometer circuit was read from minute to minute. The latter gradually increased, owing to the warming of the walls of the discharge-tube. By suddenly breaking the current and again observing the bolometer the radiation of the tube-walls was eliminated. Another method of elimination was by interposing a plate of alum about 4 millim. thick, which totally absorbed the radiation from the glass. Another source of error was the reflexion from the walls of the tube. The end of the tube opposite the bolometer was closed by a plane-parallel plate of rock-salt. This occasioned a loss by reflexion, whereas the other surfaces entailed a gain. Both were corrected by introducing a small copper box heated by steam circulation into a tube of the same construction as the discharge-tube, observing the bolometer deflexions, and repeating with the box alone.

Prof. Ångström states his main results as follows :-

1. For a given gas and a given pressure the radiation of the positive light is proportional to the intensity of the electric current.

2. For a given gas and pressure the composition of the radiation is constant and independent of the strength of current.

3. On increasing the pressure of the gas, the total radiation for a given strength of current increases as a rule, slowly at low, more rapidly at high pressures. At the same time the composition of the radiation changes, inasmuch as the ratio of the intensity of the shorter waves to the total radiation decreases. Thus the distribution of the intensity in the spectrum changes in such a manner that with diminishing pressure the intensity of radiation increases for the shorter wave-lengths.

4. The ratio between the intensity of total radiation and 
the current-work increases continuously with diminishing pressure of gas.

5. The useful optical effect of the radiation (here given by the ratio of the intensities of the radiation passing through the alum plate and the total radiation respectively) is very high for some of the gases at low pressure (about 90 per cent. for nitrogen). But the useful optical effect of the work spent is not very great (about 8 per cent. for nitrogen of 0.1 millim. pressure).

6. The intensity of total radiation must be considered as a secondary effect of the discharge, and depends upon the molecular constitution of the gas.

7. Whatever views we hold concerning the nature of the gaseous discharge, this investigation appears to confirm the hypothesis of Hittorf, E. Wiedemann, and others, that the radiation is not a pure function of the temperature of the gases, but must be regarded as anomalous ("irregular," "luminescence").

If we call "irregular" a radiation in which the spectroscopic distribution of the energy is anomalous, there are certain facts observed by Prof. Ångström which lead to the conclusion that the radiation in question is irregular. The radiation did not show any relation to the absorptive power of the gas at ordinary temperatures. Again, the radiation,which in nitrogen at 2 millim. pressure is still rich in dark rays,-rapidly changes in quality when the pressure decreases, and at 1 millim. consists almost exclusively of light radiation. Prof. Angström supposes that the radiation of the gas during electric discharge consists of two parts, one of them regular, the other irregular. With decreasing pressure the former decreases, whilst the irregular radiation increases in proportion as the motions are less obstructed by the mass of the gas. At constant pressure a certain portion of the energy in each molecule is converted into radiation; as the strength of the current increases, the number of active molecules, and hence also the radiation, increases in the same proportion as the current. The number of active molecules being relatively small, the damping effect of the rest may be taken as constant, and the composition of the radiation remains practically unaltered as the current increases. On increasing the pressure, however, the damping effect changes, the anomalous dispersion is more easily transformed into a normal one, and the radiation becomes richer in infra-red rays. A greater proportion of the energy supplied is spent in heating, and for the same current-work the total radiation decreases with increasing pressure.

Phil. Mag. S. 5. Vol. 35. No. 217. June 1893. $2 \mathrm{~N}$ 
But in view of the difficulties of the investigation, the paucity of available material, and the approximate nature of the results in this almost unexplored field, no final decision can as yet be arrived at. A tabulation and a graphic representation of the results, with diagrams of the apparatus and a full discussion of methods and corrections, will be found in the original paper.

LII. Luminous Discharges in Electrodeless Vacunm-Tules. By E. C. Rimington*.

GINCE reading a paper in conjunction with $\mathrm{Mr}$. E. W. 1 Smith on November 25th, 1892, before this Society †, on "Fxperiments in Electric and Magnetic Fields, Constant and Varying," the Author's attention has been drawn to a paper contributed by Mr. Tesla to the 'Electrical Engineer' of' New York, July 1st, 1891, in which the luminous ringshaped discharge obtained when a Leyden jar is discharged through a coil of wire surrounding an exhausted bulb is attributed to the electrostatic action of the surrounding wire, and not to the electric stress set up in the rarefied dielectric in consequence of the rapidly oscillating magnetic induction through the bulb.

As one experimental proof of this assertion Mr. Teslat gives the following experiment :- "An ordinary lamp-bulb was surrounded by one or two turns of thick copper wire, and a luminous circle excited by discharging the jar through this primary. The lamp-bulb was provided with a tinfoil coating on the side opposite to the primary, and each time the tinfoil coating was connected to the ground, or to a large object, the luminosity of the circle was considerably increased."

The author repeated this experiment with two Leyden jars arranged as in fig. 1, and found that when the spark-gap was sufficiently large to produce a bright ring when the tinfoil was not connected to earth, doing so produced no noticeable difference in the brilliancy; but that, if the discharge were faint, it was renrendered considerably brighter on making the earth connexion. Better results were, however, obtained on

Fig. 1.

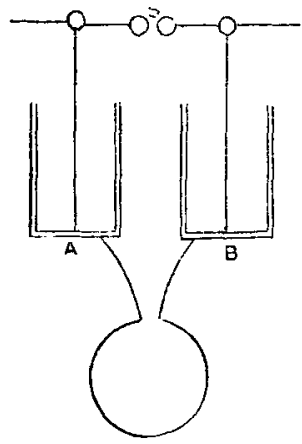

* Communicated by the Physical Society : read 4 pril $28,1893$.

† Ante, p. 98. 